Orthopäde 2022 $\cdot 51: 564-572$

https://doi.org/10.1007/s00132-021-04185-7

Angenommen: 7. Oktober 2021

Online publiziert: 30. Oktober 2021

(c) Springer Medizin Verlag $\mathrm{GmbH}$, ein Teil von Springer Nature 2021

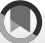

Check for
updates

\section{Individuelle Megaspacer für den zweizeitigen Wechsel infizierter Hüftendoprothesen - klinisches und funktionelles Outcome nach 2 Jahren}

\author{
Individuelle Metallendoskelett-Zementspacer (iMECS)
}

\section{Zusatzmaterial online}

Die Online-Version dieses Beitrags https:// doi.org/10.1007/s00132-021-04185-7 enthält eine tabellarische Übersicht der individuellen Pathogenlagen

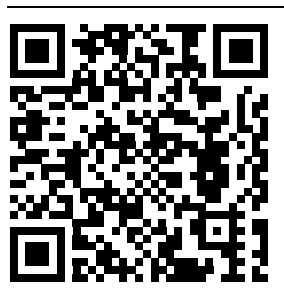

QR-Code scannen \&Beitrag online lesen
Martin Ellenrieder ${ }^{1}$ Bastian Surmann ${ }^{2}$. Andreas Enz $\cdot$ Sören Henning Toch ${ }^{1}$. Robert Lenz ${ }^{1}$. Wolfram Mittelmeier ${ }^{1}$

'Orthopädische Klinik und Poliklinik, Universitätsmedizin Rostock, Rostock, Deutschland

${ }^{2}$ Fakultät für Gesundheitswissenschaften/AG 5 Gesundheitsökonomie und Gesundheitsmanagement, Universität Bielefeld, Bielefeld, Deutschland

Zusammenfassung

Hintergrund: Große Femurdefekte bei periprothetischem Spätinfekt (PPI) nach Hüfttotalendoprothese (HTEP) erfordern oft den Einsatz großer, individueller Metallendoskelett-armierter Zementspacer (iMECS).

Ziel der Arbeit: Ziele waren die Erfassung der klinischen, radiologischen und funktionellen Ergebnisse (Harris Hip Score [HHS]) 2 Jahre nach Behandlung eines PPI unter Verwendung eines iMECS. Zentrale patientenspezifische Parameter sollten im Hinblick auf das Behandlungsergebnis ausgewertet werden.

Material und Methoden: Das Kollektiv dieser monozentrischen retrospektiven Kohortenstudie umfasste 29 Patienten. Das Follow-up zum Zeitpunkt der Nachuntersuchung (NU) betrug durchschnittlich 24,4 Monate (23,0-27,6 Monate). Als Behandlungserfolg wurde eine Infektfreiheit nach endoprothetischer Gelenkrekonstruktion gewertet. Die beiden Patientengruppen (mit Erfolg [E]/kein Erfolg [kE]) wurden hinsichtlich Geschlechterverteilung, Komorbiditätenindex nach Charlson (CCI), Anzahl früherer septischer Wechsel, Rate an polymikrobiellen und "difficult-to-treat"-Infekten verglichen.

Ergebnisse: Der durchschnittliche $\mathrm{CCl}$ im Gesamtkollektiv betrug 6,4 Punkte. Bei 23 von 29 Patienten (79\%) war eine Gelenkrekonstruktion möglich. Bei 4 Patienten war 2 Jahre nach operativer PPI-Behandlung keine NU möglich (2 verstorben, 2 nicht teilnahmefähig). Zur NU waren 17 von initial 29 Patienten infektfrei rekonstruiert mit einem durchschnittlichen HHS von 75 Punkten. Es traten keine iMECS-assoziierten revisionsbedürftigen Komplikationen auf. Zwischen den Patientengruppen war nur der initiale CCI (E: 4,1 Punkte; kE: 9,7 Punkte) signifikant unterschiedlich $(p<0,05)$. Diskussion: Bei großen Femurdefekten leisten iMECS eine sichere temporäre Stabilisierung. Die Chance auf eine erfolgreiche Gelenkrekonstruktion ist eng mit dem individuellen Komorbiditätenprofil verbunden.

Schlüsselwörter

Komorbiditäten · Infektion · Reoperation · Totaler Hüftgelenkersatz · Behandlungs-Outcome 


\section{Hinführung zum Thema}

Für den komplizierten periprothetischen Spätinfekt des Hüftgelenkes (Weichteilschäden, komplexe Keimbesiedlung) gilt der zweizeitige Wechsel als Goldstandard. Zum Erhalt der Mobilität und Weichteilspannung werden für die Interimsphase meist kommerzielle Spacer eingesetzt. Diese sind für langstreckige femorale Defekte häufig nicht ausreichend, sodass intraoperativ individuell angefertigte Spacer eingesetzt werden. Es existieren nur wenige systematisch erhobene Daten zum subjektiven und klinischen Outcome nach zweizeitigem Wechsel mit großen Individualspacern bei langstreckigem Femurdefekt.

\section{Hintergrund und Fragestellung}

Der zweizeitige Wechsel bei periprothetischem Spätinfekt (PPI) einer Hüfttotalendoprothese (HTEP) gilt als Goldstandard bei schlechten Weichteilverhältnissen, großen Knochendefekten und problematischer Keimbesiedelung („difficult-totreat", DTT-Erreger) [1-4]. Zwischen HTEPExplantation und Reimplantation erhalten intraoperativ handgefertigte oder industriell vorgefertigte Spacer aus antibiotikahaltigem Knochenzement die Weichteilspannung und die Mobilität $[2,3,5,6]$. Für den zweizeitigen HTEP-Wechsel wird eine hohe Erfolgsrate um 90\% (61-100\%) angegeben mit Infektfreiheit nach 12-96 Monaten Follow-up [7-9]. Einige Studien zum zweizeitigen septischen Wechsel schließen PPI mit großen Knochendefekten je-

\section{Abkürzungen}

CCI Komorbiditätenindex nach Charlson

$C O P D \quad$ "Chronic obstructive pulmonary disease"

DTT "Difficult-to-treat"

E Mit Erfolg

HHS Harris Hip Score

HTEP Hüfttotalendoprothese

iMECS Individueller Metallendoskelett-

armierter Zementspacer

kE Kein Erfolg

MSIS Musculoskeletal Infection Society

NU Nachuntersuchung

$O R \quad$ Odds Ratio

$P C R \quad$ Polymerase-Kettenreaktion

PPI Periprothetischer Spätinfekt

RI Reimplantation

TIA Transitorische ischämische Attacke doch aus [6, 9], sodass das Outcome dieses komplexen Patientenkollektivs unberücksichtigt bleibt. In einigen Kollektiven wurden zudem hohe Raten (bis zu 45-73\%) Spacer-assoziierter Komplikationen beobachtet $[6,10]$.

Ist der knöcherne Femurdefekt für kommerzielle Spacersysteme zu groß, fertigen einige Operateure intraoperativ individuelle Lösungen aus unterschiedlichen Materialkombinationen [11-13].

Zum Outcome sowie zu potenziellen Komplikationen nach zweizeitigem Wechsel mit großen individuellen Metallendoskelett-armierten Zementspacern (iMECS) existieren bisher jedoch kaum systematisch erhobene Daten.

Die vorliegende Arbeit hatte folgende Zielsetzungen:

- Erfassung der klinischen und funktionellen Ergebnisse 2 Jahre nach zweizeitigem Wechsel bei PPI des Hüftgelenkes unter Verwendung eines iMECS

- Analyse der Subgruppen (mit und ohne infektfreier Gelenkrekonstruktion nach 2 Jahren) auf Unterschiede bezüglich patientenspezifischer Parameter

- Erfassung von Komplikationen (abhängig und unabhängig vom iMECS)

\section{Studiendesign und Untersuchungsmethoden}

Im Rahmen dieser monozentrischen retrospektiven Kohortenstudie wurden die Daten aus den stationären Aufenthalten und den Nachuntersuchungen von Patienten mit PPI (Spätinfekt) des Hüftgelenkes analysiert. Zwischen Januar 2014 und März 2019 wurden nur Patienten eingeschlossen, bei denen ein zweizeitiger septischer HTEP-Wechsel unter Verwendung eines individuellen, mit Metallendoskelett armierten Zementspacers (iMECS) durchgeführt wurde. Die Einverständniserklärungen der Patienten und die Zustimmung der Ethikkommission wurden eingeholt (Registriernummer A 2018-0118).

\section{Patientenkollektiv und Datenerhebung}

Eingeschlossen wurden 29 Patienten im Alter von 44-85 Jahren (• Tab. 1). Aus den Vor- und Begleiterkrankungen wurde der
Komorbiditätenindex nach Charlson [14] berechnet. Die ausgewerteten Nachuntersuchungen (NU) erfolgten nach durchschnittlich 24,4 Monaten (23,0-27,6 Monate). Es wurden alle Komplikationen erfasst, die eine Verlängerung des Krankenhausaufenthaltes oder eine zusätzliche Intervention bedingten. Zur NU wurde standardisiert der Harris Hip Score (HHS) erhoben und das konventionelle Röntgen ausgewertet.

\section{Definitionen und diagnostisches Vorgehen}

Die Diagnose einer PPI wurde in dieser Kohorte gemäß den Kriterien der Musculoskeletal Infection Society (MSIS 2014) gestellt [15]. Bei klinisch nicht offensichtlichem PPI erfolgte die präoperative Stanzbiopsie (Mikrobiologie, Histologie) mit Synovialzellanalyse und Beurteilung des Membrantyps nach Krenn/Morawietz [15-17]. Bei der Explantation wurden 2 histologische Proben sowie 6 mikrobiologische Proben (14 Tage Bebrütung) entnommen [18]. Bei auswärtigen Voroperationen inklusive antimikrobieller Therapie standen zusätzlich eine $16 \mathrm{~S}$ PCR sowie eine Sonikation der Explantate zur Verfügung. Enterokokken, Pilze, Rifampicin-resistente Staphylokokken und Ciprofloxacin-resistente gramnegative Bakterien wurden als "difficult to treat"--Erreger (DTT) klassifiziert [4]. Eine Infektfreiheit zur Nachuntersuchung (NU) wurde gemäß der Delphi-basierten internationalen Konsensuskriterien festgestellt [19]. Ein Therapieerfolg wurde definiert als infektfreie endoprothetische Gelenkrekonstruktion ohne antibiotische Suppression (Erfolg: Gruppe „E“, kein Erfolg: Gruppe "kE") zum Zeitpunkt der Nachuntersuchung.

\section{Chirurgische und antimikrobielle Therapie}

Nach septischer Explantation aller Fremdmaterialien aus dem Hüftgelenk erfolgte das Debridement, die iMECS-Implantation sowie der Wundverschluss. Abhängig vom Allgemeinzustand des Patienten wurden diese Schritte in einem oder mehreren operativen Eingriffen (z.B. mit temporärer Vakuumversiegelung) durchgeführt 


\section{Originalien}

\begin{tabular}{|c|c|c|c|c|}
\hline Variable & $\begin{array}{l}\text { Alle Patienten } \\
(n=29)\end{array}$ & $\begin{array}{l}\text { Reimplantation + } \\
\text { infektfrei bei NU } \\
\text { („Erfolg“: } n=17 \text { ) }\end{array}$ & $\begin{array}{l}\text { Keine Reimplantation, } \\
\text { keine NU, nicht infektfrei } \\
\text { („,kein Erfolg“: } n=12 \text { ) }\end{array}$ & $\begin{array}{l}p \text {-Wert/ } \\
\text { Odds Ratio } \\
\text { (OR) }\end{array}$ \\
\hline Alter bei iMECS (Jahre) & $70,6 \pm 9,9$ & $69,1 \pm 9,8$ & $72,7 \pm 10,1$ & $p=0,36$ \\
\hline Geschlecht (männl./weibl.) & $M: W=18: 11$ & $\mathrm{M}: \mathrm{W}=12: 5$ & $M: W=6: 6$ & OR: 2,40 \\
\hline Body-Mass-Index $\left(\mathrm{kg} / \mathrm{m}^{2}\right)$ & $31,2 \pm 6,3$ & $31,9 \pm 5,6$ & $30,2 \pm 7,5$ & $p=0,53$ \\
\hline Revisionen nach Primär-HTEP $(n)$ & $1,8 \pm 1,9$ & $1,7 \pm 1,9$ & $2,0 \pm 1,7$ & - \\
\hline Frühere septische Wechsel (Anteil) & $13 / 29(45 \%)$ & $7 / 17(41 \%)$ & $6 / 12(50 \%)$ & OR: 1,43 \\
\hline Charlson Komorbiditätsindex (CCI) & $6,4 \pm 3,6(1-14)$ & $4,1 \pm 1,9(1-10)$ & $9,7 \pm 2,7(6-14)$ & $p<0,05^{*}$ \\
\hline $\mathrm{CCl} \geq 7$ & $12 / 29(41 \%)$ & $1 / 17(6 \%)$ & $11 / 12(92 \%)$ & OR: $176^{*}$ \\
\hline Polymikrobieller Infekt & $12 / 29(41 \%)$ & $6 / 17(35 \%)$ & $6 / 12(50 \%)$ & OR: 1,83 \\
\hline DTT-Erreger & $11 / 29(38 \%)$ & $6 / 17(35 \%)$ & $5 / 12(42 \%)$ & OR: 1,31 \\
\hline Femorale Defektstrecke (mm) & $212 \pm 71$ & $205 \pm 71$ & $222 \pm 73$ & - \\
\hline iMECS einzeitig (Anteil) & $5 / 29(17 \%)$ & $3 / 17(18 \%)$ & $2 / 12(16 \%)$ & - \\
\hline Operationsdauer (iMECS einzeitig) (min) & $148 \pm 34$ & 133 & 170 & - \\
\hline Operationsdauer (iMECS mehrzeitig) (min) & $117 \pm 30$ & 112 & 124 & - \\
\hline Spacermaterialkosten (Euro) & 1249 & 1227 & 1284 & - \\
\hline KH-Aufenthalt (Sanierung) (d) & $34,8 \pm 23,9$ & $33,7 \pm 19,9$ & $36,5 \pm 28,1$ & - \\
\hline Anteil Patienten mit Rekonstruktion & 23 von 29 & 17 von 17 & 6 von 12 & - \\
\hline Spacerintervall (falls rekonstruiert) (d) & $88,8 \pm 31,8$ & $89,6 \pm 26,5$ & $86,3 \pm 38,7$ & - \\
\hline KH-Aufenthalt (Rekonstruktion) (d) & $19,8 \pm 12,3$ & $18,9 \pm 12,8$ & $22,2 \pm 9,4$ & - \\
\hline Operationsdauer (Rekonstruktion) (min) & $154 \pm 32$ & $157 \pm 33$ & $147 \pm 30$ & - \\
\hline Harris Hip Score (HHS) & - & $75 \pm 16$ & Entfällt & - \\
\hline \multicolumn{5}{|c|}{$\begin{array}{l}\text { Angegeben sind Mittelwerte und } 1 \text { Standardabweichung oder Prozentangaben } \\
D T T \text { "difficult to treat", HTEP Hüfttotalendoprothese, iMECS individueller Metallendoskelett-armierter Zementspacer, KH-Aufenthalt Dauer der stationären } \\
\text { Behandlung, } N U \text { Nachuntersuchung inklusive HHS-Erhebung } \\
\text { *Statistisch signifikant }(p \leq 0,05)\end{array}$} \\
\hline
\end{tabular}

\begin{tabular}{|c|c|c|}
\hline Keim & $\begin{array}{l}\text { Anzahl der } \\
\text { Isolate }\end{array}$ & $\begin{array}{l}\text { Anteil } \\
(\%)\end{array}$ \\
\hline $\begin{array}{l}\text { Koagulase-negative } \\
\text { Staphylokokken }\end{array}$ & 17 & 31 \\
\hline S. aureus & 6 & 11 \\
\hline Candida/Pilze & 6 & 11 \\
\hline Enterokokken & 5 & 9 \\
\hline $\begin{array}{l}\text { Streptokokken (diver- } \\
\text { se) }\end{array}$ & 5 & 9 \\
\hline Cutibacterium & 5 & 9 \\
\hline E. coli & 4 & 7 \\
\hline S. hominis/capitis & 3 & 5 \\
\hline Andere & 4 & 7 \\
\hline
\end{tabular}

(• Tab. 1). Das iMECS-Konstrukt (• Abb. 1) bestand aus einem Metallendoskelett (z. B. DePuy Synthes GmbH, Oberdorf, Schweiz) und Knochenzement. Als Standard diente dabei COPAL $^{\circledR} \mathrm{G}+\mathrm{C}$ (Heraeus Medical $\mathrm{GmbH}$, Wehrheim, Deutschland). Gemäß
Erregerspektrum und Antibiogramm wurden dem Zement testgerecht geeignete Antiinfektiva (thermostabil, hydrophil) bis $4 \mathrm{~g}$ pro $40 \mathrm{~g}$ Zement zugesetzt [1, 20]. Dies wurde bei entsprechend sensiblen Erregern durch manuelles Zumischen von $2 \mathrm{~g}$ Vancomycin erreicht. Alternativ wurde der Knochenzement zusätzlich mit Gentamicin, Linezolid, Ciprofloxacin oder Voriconazol beladen mittels fraktionierter Zugabe und manuellem Anrühren $[1,2]$. Nach Abschluss der chirurgischen Sanierung durch iMECS-Implantation mit Wundverschluss erfolgte eine testgerechte Antibiotikagabe für weitere 4 Wochen, bei Infekt mit DTT-Erregern für 6 Wochen. Wir strebten grundsätzlich ein Spacerintervall von 8 Wochen an [18]. Die Entscheidung über die Möglichkeit und den Zeitpunkt der Reimplantation wurde in diesem komplexen Patientenkollektiv jedoch individuell abgewogen. Ergaben sich anhand der mikrobiologischen/histologischen Proben aus der Reimplantation Hinweise für eine
Infektpersistenz, wurde die antimikrobielle Therapie von 4 auf 8 Wochen verlängert.

\section{Statistik}

Die deskriptive Darstellung der Daten erfolgte als Mittelwert mit Standardabweichung (MW $\pm 1 S D$ ), ggf. zusätzlich auch der Minimal- und Maximalwerte. Unterschiede zwischen den Gruppen wurden für metrische Variablen bei zugrundeliegender Normalverteilung mittels $\mathrm{t}$-Test und bei nicht normalverteilten Daten mittels Mann-Whitney-U-Test geprüft. Für kategoriale Faktoren wurde jeweils die Odds Ratio (OR) berechnet, deren Signifikanz mit dem exakten Test nach Fisher bewertet wurde. Grundsätzlich wird $p<0,05$ als statistisch signifikant verstanden. Die statistische Auswertung erfolgte mit SPSS 27.0 (IBM Deutschland GmbH, Ehningen, Deutschland) und R (Version 3.0.6). 


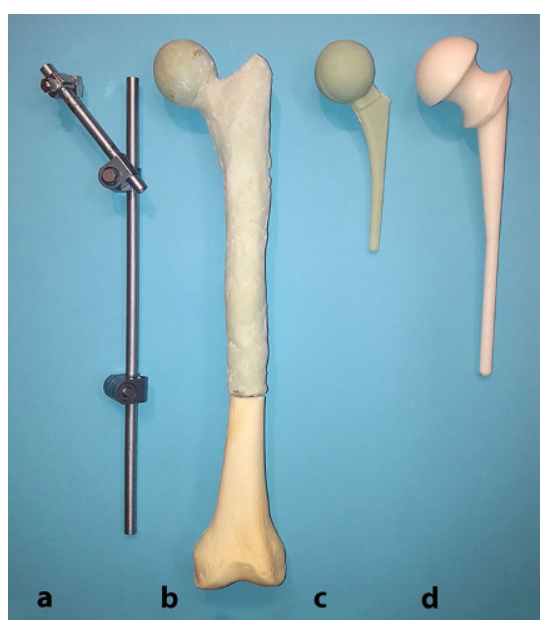

Abb. $1 \Delta$ Mit einem Metallendoskelett (a) ausgestatteter Megaspacer (b) (iMECS [individueller Metallendoskelett-armierter Zementspacer]). Kommerzielle Spacer ([c] StageOne ${ }^{\mathrm{TM}}$, Zimmer Biomet, Warsaw, IN, USA; (d) Spacer ${ }^{\circledR}$ GXL, Merete GmbH, Berlin, Deutschland) sind für langstreckige Femurdefekte nicht ausreichend

\section{Ergebnisse}

Zwischen Januar 2014 und März 2019 führten wir an unserem EndoProthetikZentrum bei 102 Patienten einen zweizeitigen septischen HTEP-Wechsel durch, davon bei 29 Patienten (28\%) mit einem iMECS.

\section{Sanierungsphase und Spacerinter- vall}

Alle 29 Patienten erfüllten die MSIS-Kriterien für einen PPI [15] und hatten einen positiven Keimnachweis für mindestens einen Erreger ( Tab. 1 und 2). Bei zwei Patienten mit auswärtiger Voroperation (Exploration/septische Explantation) und Antibiotikatherapie erfolgte der relevante Erregernachweis ausschließlich mittels PCR (jeweils $S$. aureus). In beiden Fällen bestätigte sich die Infektion auch histologisch (Typ-II-Membran) bei der Explantation. Die femorale Defektstrecke von der Gelenklinie bis zum proximalsten tragfähigen Punkt der Femurdiaphyse betrug im Mittel $212 \mathrm{~mm}$ (• Tab. 1). Nur bei $17 \%$ der Patienten wurde die chirurgische Infektsanierung einschließlich iMECS-Implantation und Wundverschluss mittels eines einzigen operativen Eingriffes durchgeführt. Zehn der 23 Patienten mit Gelenkrekonstruktion erhielten wäh-

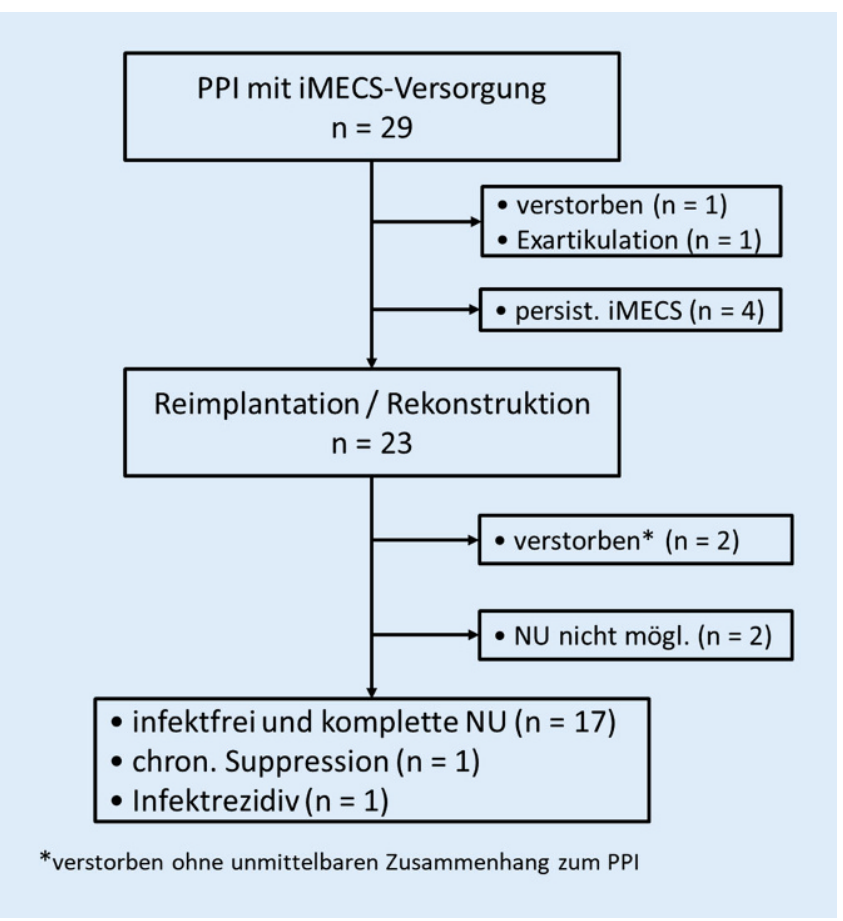

Abb. $2 \triangleleft$ Flowchart zum Behandlungsverlauf der initial eingeschlossenen 29 Patienten. HHS Harris Hip Score, iMECS individueller Metallendoskelett-armierter Zementspacer, $N U$ Nachuntersuchung, $P P I$ periprothetischer Spätinfekt rend der Interimsphase eine von 4 auf 6 Wochen verlängerte Antibiotikagabe bei DTT-Infekt. Aufgrund der erforderlichen Spacerintervalle von durchschnittlich 2,9 Monaten (- Tab. 1) erfolgte vor der Reimplantation die Untersuchung (Klinik, Labor, Stanzbiopsie) hinsichtlich Infektzeichen mit mindestens 2 Wochen Abstand zur letzten Antibiotikagabe [17].

Bei 6 von 29 Patienten (21\%) wurde der stationäre Aufenthalt der Sanierungsphase durch Komplikationen ohne erkennbaren Zusammenhang zum iMECS verlängert:

- Wundrevisionen (2 Patienten)

- Hämatomausräumung bei therapeutischer Antikoagulation (1 Patient)

- postoperatives Delir (2 Patienten)

- gastrointestinale Blutung mit endoskopischer Intervention (1 Patient)

Eine multimorbide Patientin mit bereits initial septischem Krankheitsbild (polymikrobielle DTT-Infektion) und schwerer erworbener Gerinnungsstörung verstarb noch während des Sanierungszyklus (• Tab. 3). Im Rahmen eines Suizidversuchs kam es bei einem Patienten zur Spacerluxation (geschlossene Reposition) und zur periimplantären proximalen Femurfraktur ohne Instabilität (konservativ behandelt). Spacer-assoziiert trat bei zwei Patienten $(6,9 \%)$ mit azetabulären Defekten eine
Subluxation des iMECS nach kranial auf, jedoch ohne Revisionsbedürftigkeit.

\section{Rekonstruktionsphase und Follow-up}

Bei insgesamt 6 Patienten war eine Reimplantation nicht möglich oder nicht indiziert (-Abb. 2; - Tab. 3). Einen iMECS erhielten noch 4 dieser 6 Patienten, ein Patient verstarb (Sepsis), ein weiterer wurde aufgrund der Gesamtsituation exartikuliert (- Abb. 2). Bei 2 von 4 Patienten mit persistierendem iMECS wurde aufgrund der palliativen Situation (1x onkologisch, 1x internistisch) keine Reimplantation avisiert. Bei den beiden anderen Patienten (multimorbide, pflegebedürftig) mit persistierendem iMECS war eine Verbesserung der Mobilität auch durch ein definitives Implantat nicht zu erwarten, somit war die Reimplantation nicht indiziert (- Tab. 3). Alle 4 Patienten mit persistierendem iMECS verblieben nach Absetzen der Antibiotika infektfrei. Für die 23 Patienten mit Gelenkrekonstruktion ( $n=23)$ wurde bei 20 Patienten (87\%) femoral ein modulares Revisionssystem eingesetzt ( $\mathbf{A b b}$. 3), bei 3 Patienten (13\%) ein langer Monoblockstiel. Bei 2 dieser 23 Patienten wurde die Antibiotikagabe auf 8 Wochen verlängert bei Infektzeichen 
Tab. 3 Übersicht der Patienten aus der Gruppe „kE“ für die keine oder keine vollständige 2-Jahres-Untersuchung vorlag. Ordnung der Studienausfälle nach Zeitabstand (aufsteigend) zur septischen Explantation korrespondierend zu $\bullet \mathbf{A b b}$.

\begin{tabular}{|c|c|c|c|c|}
\hline $\begin{array}{l}\text { Geschlecht/ } \\
\text { Alter; (CCI) }\end{array}$ & Besondere Komorbiditäten & Zeitpunkt & $\begin{array}{l}\text { Grund Studien- } \\
\text { ausfall }\end{array}$ & $\begin{array}{l}\text { Letzter Status/Zusatzinformation Outcome bis } \\
2 \text { Jahre nach iMECS }\end{array}$ \\
\hline W/73 J.; (11) & Niereninsuffizienz, Leberzirrhose & Vor RI & Verstorben & $\begin{array}{l}\text { Erworbene Koagulopathie, Nieren- und konsekutives } \\
\text { Multiorganversagen }\end{array}$ \\
\hline W/81 J.; (12) & $\begin{array}{l}\text { Z.n. Apoplex (Paresen), Kolonkarzinom, } \\
\text { Herzinsuffizienz, D. mellitus }\end{array}$ & Vor RI & Exartikulation & Schwere Demenz, Exartikulation (infektfrei) \\
\hline M/68 J.; (14) & $\begin{array}{l}\text { Multipel metastasiertes Brochialkarzinom } \\
\text { vor PPI }\end{array}$ & Keine RI & $\begin{array}{l}\text { Persistierender } \\
\text { Spacer }\end{array}$ & $\begin{array}{l}\text { Metastasiertes Bronchialkarzinom (verstorben } \\
3 \text { Mon. nach iMECS) }\end{array}$ \\
\hline W/45 J.; (12) & Adipositas, KHK, Diabetes, Z.n. Sepsis & Keine RI & $\begin{array}{l}\text { Persistierender } \\
\text { Spacer }\end{array}$ & Myokardinfarkt (verstorben 9 Mon. nach iMECS) \\
\hline M/63 J.; (11) & $\begin{array}{l}\text { Dialysepflichtig, Z.n. Nieren-Pankreas- } \\
\text { Transplantation }\end{array}$ & Keine RI & $\begin{array}{l}\text { Persistierender } \\
\text { Spacer }\end{array}$ & $\begin{array}{l}\text { Pflegebedürftig, schlechter Allgemeinzustand, De- } \\
\text { menz, mobil mit Rollstuhl }\end{array}$ \\
\hline W/84 J.; (7) & $\begin{array}{l}\text { Synchron: Spondylodeseinfekt, Z.n. kardia- } \\
\text { ler Dekompensation }\end{array}$ & Keine RI & $\begin{array}{l}\text { Persistierender } \\
\text { Spacer }\end{array}$ & $\begin{array}{l}\text { Pflegebedürftig, später SARS-CoV-2-Infektion, Rolla- } \\
\text { tor mit Hilfe }\end{array}$ \\
\hline W/75 J.; (6) & $\begin{array}{l}\text { Herzinsuffizienz, chronische Immobilität } \\
\text { (schwere SKS) }\end{array}$ & $\begin{array}{l}\text { 4 Monate } \\
\text { nach RI }\end{array}$ & Infektrezidiv & $\begin{array}{l}\text { Schlechte Weichteilverhältnisse, Immobilität, Exarti- } \\
\text { kulation im Verlauf }\end{array}$ \\
\hline W/85 J.; (12) & $\begin{array}{l}\text { Z.n. Apoplex/Hemiparese, Niereninsuffizi- } \\
\text { enz, D. mellitus }\end{array}$ & $\begin{array}{l}14 \text { Mon. } \\
\text { nach RI }\end{array}$ & Verstorben & Re-Apoplex mit Todesfolge \\
\hline $\mathrm{M} / 58 \mathrm{~J} . ;(7)$ & Hepatitis B, Dialysepflicht & $\begin{array}{l}16 \text { Mon. } \\
\text { nach RI }\end{array}$ & Verstorben & Sepsis mit Todesfolge nach Shuntinfekt \\
\hline $\mathrm{M} / 80 \mathrm{~J} . ;(7)$ & Z.n. Apoplex mit Residuen, Z.n. Sepsis, KHK & $\begin{array}{l}\text { Zur NU } \\
\text { nach 2J }\end{array}$ & $\begin{array}{l}\text { NU nicht mög- } \\
\text { lich }\end{array}$ & Pflegebedürftig, progressive Demenz, immobil \\
\hline M/83 J.; (10) & $\begin{array}{l}\text { Z. n. multiplen Embolien, COPD, Z.n. mehr- } \\
\text { fache TIA/Apoplex }\end{array}$ & $\begin{array}{l}\text { Zur NU } \\
\text { nach 2J }\end{array}$ & $\begin{array}{l}\text { NU nicht mög- } \\
\text { lich }\end{array}$ & $\begin{array}{l}\text { Pflegebedürftig, Herzinsuffizienz, mobil am Rollator } \\
\text { mit Hilfe }\end{array}$ \\
\hline
\end{tabular}

BMI Body-Mass-Index in $\left(\mathrm{kg} / \mathrm{m}^{2}\right)$, CCI Charlson Komorbiditäten Index, COPD „chronic obstructive pulmonary disease“, HHS Harris Hip Score, iMECS individueller Metallendoskelett-armierter Zementspacer, KHK koronare Herzkrankheit, $M$ männlich, NU Nachuntersuchung, PPI periprothetischer Spätinfekt, RI Reimplantation, SKS Spinalkanalstenose, TIA transitorische ischämische Attacke, $W$ weiblich

(mikrobiologisch/histologisch) aus der Reimplantation.

Bis zur NU wurde aufgrund einer Rezidivluxation bei einem Patienten ein längeres Diaphysenmodul des Femurersatzes (plus $10 \mathrm{~mm}$ ) implantiert, ein adipöser Patient (BMI 35) benötigte einen aseptischen Stielwechsel. Von initial 29 Patienten verblieben 19 Patienten zur 2-Jahres-NU, davon 17 Patienten mit infektfreier Gelenkrekonstruktion und ohne radiologische Zeichen einer Infektion (Osteolysen) oder Implantatlockerung. Ein Patient erhielt bei Infektrezidiv einen erneuten septischen Wechsel. Ein Patient mit u.a. schwerer Alkoholenzephalopathie erhielt eine chronische Suppressionstherapie (• Abb. 2). Die Datenanalyse zeigte bezüglich der untersuchten patientenspezifischen Parametern nur für den $\mathrm{CCl}$ einen signifikanten Unterschied zwischen den Gruppen „E“ und „kE" (- Tab. 3). Die Patienten mit rekonstruiertem Gelenk erzielten einen Harris Hip Score von durchschnittlich $75 \pm 16$ Punkten (- Tab. 1).

\section{Diskussion}

Die Erfolgsraten von über $90 \%$ nach zweizeitigem Wechsel bei PPI des Hüftgelenkes [8] müssen kritisch beurteilt werden mit Blick auf die tatsächliche Rate an Reimplantationen, die Komorbiditäten und die femorale Defektstrecke eines Studienkollektivs [5, 7, 21, 22]. Die vorliegende Arbeit war fokussiert auf ein mit erheblichen Komorbiditäten belastetes Patientenkollektiv mit PPI des Hüftgelenkes und großer femoraler Defektstrecke. Kommerzielle Spacer bieten für große femorale Defekt entweder eine zu geringe Verankerungsstrecke oder haben als Sonderimplantat mehrere Wochen Fertigungszeit [23].

In unserem Kollektiv lag der mittlere CCI bei 6,4 Punkten und bei $45 \%$ der Patienten betrug der $\mathrm{CCl} \geq 6$ Punkte. Ein $\mathrm{CCl} \geq 7$ Punkte war in der Subgruppe „"KE" signifikant häufiger als in Subgruppe „E" mit infektfreier Gelenkrekonstruktion zur NU. Einen $\mathrm{CCl} \geq 6$ Punkte fanden Corona et al. nur bei $6,9 \%$ ihrer Patienten $(n=162)$ mit PPI des Hüft- und Kniegelenkes [22]. Gomez et al. errechneten lediglich einen mittleren $\mathrm{CCl}$ von 4,2 Punkte für ihr Kollektiv $(n=178)$ mit PPI bei HTEP [21]. Ein höherer CCI war ebenfalls signifikant assoziiert mit einer niedrigeren Rate an Reimplantationen [21]. Zusammenfassend gefährdet somit ein $\mathrm{CCl}$ von 7-10 Punkten eine spätere erfolgreiche Gelenkrekonstruktion. Bei einem $\mathrm{CCl}>10$ Punkte dürfte eine Reimplantation absoluten Einzelfällen vorbehalten bleiben. Im vorliegenden Kollektiv fand sich zwischen den Subgruppen $(E, k E)$ kein Unterschied bezüglich Geschlechterverteilung und BMI. Bei Gomez et al. war ein höherer BMI mit einer größeren Zahl erforderlicher Sanierungsoperationen, aber nicht mit einem schlechteren Behandlungserfolg assoziiert [21]. Für polymikrobielle PPI und DTT-Erreger fanden wir eine tendenzielle Häufung in der Gruppe „kE“. Für PPI mit der Subgruppe gramnegativer Bakterien ist eine geringere Reimplantationsrate beschrieben [21, 22]. Grundsätzlich erschweren DTT-Erreger die Infektsanierung aufgrund ihrer Resistenz gegen biofilmaktive und gut gewebegängige Antibiotika [1, 4]. Dennoch können vergleichbare Erfolgsquoten erzielt werden wie bei der Therapie 

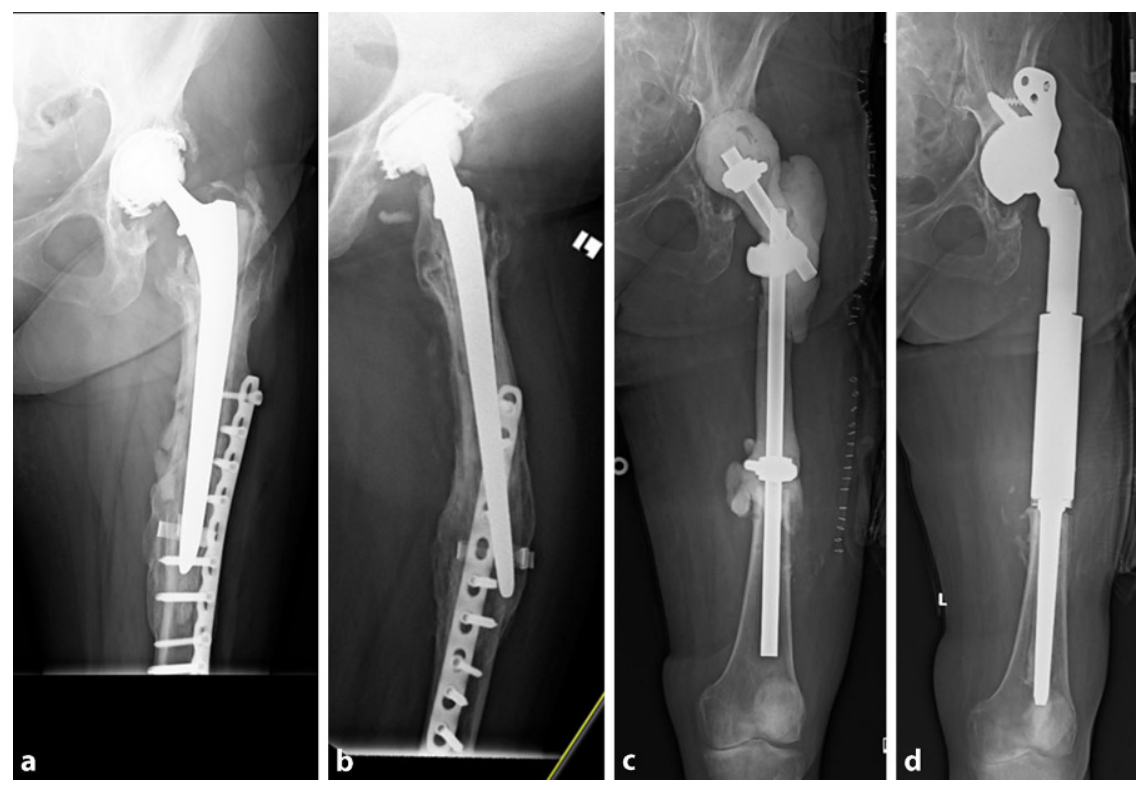

Abb. $3 \Delta$ Spätinfekt bei einliegender Hybrid-Hüftendoprothese mit Z. n. Stielwechsel und Osteosynthese einer diaphysären Femurfraktur alio loco $(\mathbf{a}, \mathbf{b})$. Nach kompletter Fremdmaterialentfernung und Debridement einliegender iMECS (individueller Metallendoskelett-armierter Zementspacer) (c). Spätere Rekonstruktion mit modularen Revisionssystemen für Pfanne und Femur (d)

von nicht-DTT-PPI [9, 21, 22]. Von zentraler Bedeutung sind ein konsequentes Debridement und eine Anpassung des chirurgischen Behandlungsalgorithmus (z.B. längere Interimsphase $>4$ bis 8 Wochen bei DTT-Erregern) $[1,3,4,9]$. Ein interdisziplinärer Behandlungsansatz (orthopädische Chirurgie, Infektiologie, Mikrobiologie, plastische Chirurgie) wird empfohlen $[1-3,9]$. Aus unserer Sicht sollte frühzeitig auch die Abstimmung mit der Anästhesie/Intensivmedizin und Schmerztherapie erfolgen sowie bei Bedarf eine psychologische Mitbetreuung der Patienten stattfinden.

Die optimale Strategie zur Antibiotikatherapie bei der zweizeitigen PPI-Sanierung wird diskutiert [24]. Izakovicova et al. erarbeiteten jedoch Empfehlungen zur lokalen und systemischen Antibiotikaapplikation für die PPI-Sanierung [1]. Bei günstiger Befundlage werden zunehmend kürzere Spacerintervalle ( $<6$ Wochen) bevorzugt sowie eine durchgehende Antibiotikagabe von 6-12 Wochen nach Reimplantation [1-4, 9]. Mit Blick auf die Nebenwirkungen muss eine Gesamtdauer der antibiotischen Therapie von über 12 Wochen individuell und kritisch geprüft werden [1, 3]. Für den Zeitraum nach der Reimplantation werden biofilmaktive Antibiotika (z. B. Ciprofloxacin, Rifampicin) empfohlen, wobei Rifampicin erst bei geschlossenen Wundverhältnissen und nicht als Monotherapie (Resistenzentwicklung) gegeben werden sollte [1, 3, 4]. Im vorliegenden Kollektiv betrug das Spacerintervall durchschnittlich 2,9 Monate aufgrund der Weichteilsituation, der Komorbiditäten und z.T. multilokulärer Infektherde (Wirbelsäule, andere Gelenke). Dies korreliert mit den Intervallen (2-11,7 Monate) anderer Arbeiten zu PPI mit großen femoralen Defekten und Individualspacern [12, 13]. Das iMECS-Konstrukt erwies sich dabei als mechanisch bruchstabil und lediglich in $6,9 \%$ der Fälle kam es zu einer Subluxation bei azetabulären Defekten (ohne Revisionsbedürftigkeit). Die Komplikationsraten nach Versorgung selbst relativ kleiner Defekte mit handgefertigten und kommerziellen Spacern liegen mit bis zu $26-73 \%$ meist deutlich höher [5, $6,10]$. Spacerbrüche, periimplantäre Frakturen und revisionsbedürftige Luxationen sind die wesentlichen Gründe für deren mechanisches Versagen $[5-7,10,13,25]$. Das massive Endoskelett des iMECS-Konstruktes stabilisiert auch langstreckige Versorgungen. Durch die modulare Bauart können die Empfehlungen zu einer suffizienten endofemoralen Verankerung und einer günstigen "head-neck-ratio" realisiert werden [5]. Die In-situ-Verbindung
(Klemmbacke) unter maximaler Distraktion zwischen dem bereits azetabulär positionierten Kopf-Hals-Segment und dem diaphysären Anteil ermöglicht die optimale Einstellung der Weichteilspannung vor der kompletten Zementarmierung. Daraus resultiert eine hohe Luxationssicherheit des iMECS. Der komplikationsträchtige Einsatz zusätzlicher azetabulärer Zementschalen [6] kann so vermieden werden. Allerdings ist der zeitliche und finanzielle Aufwand (•Tab. 1) zur intraoperativen Herstellung großer Individualspacer erheblich [12]. Dabei sollte das manuelle Beimischen von Antibiotika zum Spacerzement unter möglichst geringer mechanischer Belastung des Gemisches erfolgen, um das Entstehen von Abriebpartikeln aus dem Mischsystem zu vermeiden [26]. Mit liegendem antibiotikahaltigem Spacer ist die alleinige Synoviaanalyse nicht verlässlich bezüglich der Infektdiagnostik vor der Reimplantation [27].

Bei ausgedehnten HTEP-PPI wie im vorliegenden Kollektiv kann eine infektfreie Rekonstruktion nicht regelhaft erreicht werden. Nach iMECS wurde in $79 \%$ der Fälle (23 von 29) das Gelenk endoprothetisch rekonstruiert, bei insgesamt 3/29 Patienten (10,3\%) konnte die Infektion bis zur NU nicht beherrscht werden. In der Literatur reicht die Rate an persistierenden und Reinfektionen nach hüftnahem PPI von 11,5-33\% [11, $13,28]$. Sancineto et al. konnten in ihrer Fallserie mit individuellen Megaspacern nur bei 7 von 11 (64\%) Patienten eine Gelenkrekonstruktion vornehmen [12]. In einigen Kollektiven wurde der Hüftspacer in bis zu 36-68\% der Fälle als endgültiges Implantat belassen [12, 29]. Für Spacer als Dauerimplantate werden hohe Versagensraten von bis zu $73 \%$ beschrieben [30]. In der vorliegenden Studie verblieb der iMECS nur in $14 \%$ der Fälle als Dauerimplantat, jedoch ohne mechanisches Versagen und ohne Infektzeichen.

Bei liegendem/persistierendem Spacer erzielen Patienten oft nur eine unbefriedigende Hüftfunktion gemessen am HHS von 37-60 Punkten $[6,25]$. Für Patienten mit erheblichen Komorbiditäten und reduziertem Mobilitätsanspruch ist dies jedoch meist akzeptabel, solange keine Spacerkomplikation auftritt [29]. Auch nach endoprothetischer Hüftgelenkrekon- 
struktion sind die funktionellen Ergebnisse selbst bei kleinen femoralen Defekten variabel und reichen im Durchschnitt von unbefriedigend bis bestenfalls gut (HHS: 64-89 Punkte) [6, 8]. Übereinstimmend mit Artiaco et al. [13] verzeichneten wir für die endoprothetische Hüftrekonstruktion bei ausgedehnten femoralen Defekten nach 2 Jahren eine befriedigende Gelenkfunktion (HHS: 75 Punkte).

\section{Limitationen}

- Retrospektive monozentrische Studie ohne Kontrollgruppe

- relativ kleines Patientenkollektiv,

- Follow-up nur 2 Jahre.

\section{Ausblick}

$\mathrm{Ob}$, und vor allem wie der $\mathrm{CCl}$ und ggf. andere patientenindividuelle Faktoren als Instrumente für die frühzeitige Festlegung der Therapiestrategie einzusetzen sind, wäre anhand größerer, kontrollierter prospektiver Studien zu prüfen. Das eingesetzte iMECS-Konstrukt erwies sich als mechanisch stabil und komplikationsarm. Dessen Eigenschaften (Modularität, Individualisierbarkeit, Geometrie) wären die optimale Grundlage für die Entwicklung eines kommerziellen, ständig verfügbaren Baukastensystems.

\section{Fazit für die Praxis}

- Individuelle Metallendoskelett-Zementspacer (iMECS) sind eine mechanisch stabile temporäre Lösung für langstreckige Femurdefekte beim zweizeitigen Hüftendoprothesen(HTEP)-Wechsel.

- Bei der intraoperativen Anfertigung des iMECS können einige der bekannten Gründe für ein mechanisches Versagen von Spacern gezielt adressiert werden.

- In einem Kollektiv mit schweren Komorbiditäten und ausgedehnten femoralen Defekten nach periprothetischem HTEPSpätinfekt ist eine Gelenkrekonstruktion nicht immer möglich.

- Der Komorbiditätenindex (CCI) war in der Patientengruppe ohne erfolgreiche Gelenkrekonstruktion signifikant höher als bei Patienten mit infektfreier HTEP-Reimplantation nach 2 Jahren.

- Eine bessere Verfügbarkeit kommerzieller modularer Spacersysteme für große Femurdefekte wäre wünschenswert.
Korrespondenzadresse

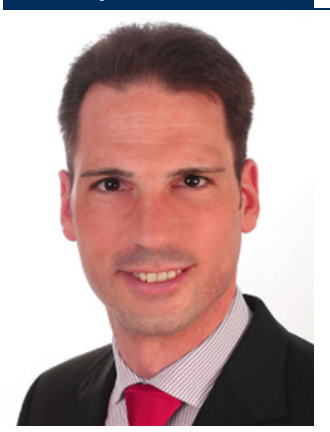

PD Dr. med. habil. Martin Ellenrieder Orthopädische Klinik und Poliklinik, Universitätsmedizin Rostock Doberaner Straße 142, 18057 Rostock, Deutschland martin.ellenrieder@med.uni-rostock.de

\section{Einhaltung ethischer Richtlinien}

Interessenkonflikt. M. Ellenrieder, B. Surmann, A. Enz, S.H. Toch, R. Lenz und W. Mittelmeier geben an, dass kein Interessenkonflikt besteht.

Alle beschriebenen Untersuchungen am Menschen oder an menschlichem Gewebe wurden mit Zustimmung der zuständigen Ethikkommission, im Einklang mit nationalem Recht sowie gemäß der Deklaration von Helsinki von 1975 (in der aktuellen, überarbeiteten Fassung) durchgeführt (Registriernummer A 2018-0118). Von allen beteiligten Patienten liegt eine Einverständniserklärung vor.

\section{Literatur}

1. Izakovicova P, Borens O, Trampuz A (2019) Periprostheticjoint infection: current concepts and outlook. EFORT Open Rev 4(7):482-494

2. Mühlhofer HML, Feihl S, Suren C et al (2020) Implantatassoziierte Gelenkinfektionen. Orthopade 49(3):277-286

3. Lehner B, Omlor GW, Schwarze M (2020) Periprothetische Früh- und Spätinfektionen: Neueste Entwicklungen, Strategien und Behandlungsalgorithmen. Orthopade 49(8):648-659

4. Zimmerli W, Trampuz A, Ochsner PE (2004) Prosthetic-joint infections. N Engl J Med 351(16):1645-1654

5. Jones CW, Selemon N, Nocon A et al (2019) The influence of spacer design on the rate of complications in two-stage revision hip arthroplasty. J Arthroplasty34(6):1201-1206

6. YangFS, LuYD, WuCTetal(2019) Mechanicalfailure of articulating polymethylmethacrylate (PMMA) spacers in two-stage revision hip arthroplasty: the risk factors and the impact on interim function. BMC Musculoskelet Disord 20(1):372

7. Rava A, Bruzzone M, Cottino U et al (2019) Hip spacers in two-stage revision for periprosthetic joint infection: a review of literature. Joints 7(2):56-63

8. Lee WY, Hwang DS, Kang C et al (2017) Usefulness of prosthesis made of antibiotic-loaded acrylic cement as an alternative implant in older patients with medical problems and periprosthetic hip infections: A 2- to 10-Year Follow-Up Study. JArthroplasty 32(1):228-233

9. Akgün D, Müller M, Perka C et al (2019) High cure rate of periprosthetic hip joint infection with multidisciplinary team approach using standardized two-stage exchange. J Orthop Surg Res 14(1):78

10. Erivan R, Lecointe T, Villatte $G$ et al (2018) Complications with cement spacers in 2-stage treatment of periprosthetic joint infection on total hip replacement. Orthop Traumatol Surg Res 104(3):333-339

11. Müller M, Winkler T, Märdian S et al (2019) The worst-case scenario: treatment of periprosthetic femoral fracture with coexistent periprosthetic infection-a prospective and consecutive clinical study. Arch Orthop Trauma Surg 139(10):1461-1470

12. Sancineto CF, Pereira DM et al (2019) Preliminary outcomes of proximal femur megaspacers. Arthroplast Today 5(2):164-171

13. Artiaco S, Boggio F, Colzani G et al (2013) Megaprostheses in the revision of infected total hip arthroplasty. Clinical series and literature review. Bull Hosp Jt Dis 73(4):229-232

14. Charlson ME, Pompei P, Ales KL et al (1987) A new method of classifying prognostic comorbidity in longitudinal studies: development and validation. JChronic Dis 40(5):373-383

15. Parvizi J, Gehrke T (2014) International Consensus Group on Periprosthetic Joint Infection. Definition of periprosthetic joint infection. J Arthroplasty 29(7):1331

16. Krenn V, Morawietz L, Perino G et al (2014) Revised histopathological consensus classification of joint implant related pathology. Pathol Res Pract 210(12):779-786

17. Enz A, Becker J, Warnke P et al (2020) Increased diagnostic certainty of periprosthetic joint infections by combining microbiological results with histopathological samples gained via a minimally invasive punching technique. J Clin Med 9:3364

18. Ellenrieder M, Lenz R, Haenle M et al (2011) Twostage revision of implant-associated infections after total hip and knee arthroplasty. GMS Krankenhhyg Interdiszip 6(1):Doc17

19. Diaz-Ledezma C, Higuera CA, Parvizi J (2013) Success after treatment of periprosthetic joint infection: a Delphi-based international multidisciplinary consensus. Clin Orthop Relat Res 471(7):2374-2382

20. Kühn KD, Renz N, Trampuz A (2017) Lokale Antibiotikatherapie. Unfallchirurg 120(7):561-572

21. Gomez MM, Tan TL, Manrique J et al (2015) The fate of spacers in the treatment of periprosthetic joint infection. J Bone Joint Surg Am 97(18):1495-1502

22. Corona PS, Vicente M, Carrera L et al (2020) Current actual success rate of the two-stage exchange arthroplasty strategy in chronic hip and knee periprosthetic joint infection. Bone Joint J 102B(12):1682-1688

23. https://www.tecres.it/en/products/orthopaedics/ custom-made-service/custom-made-solution. Zugegriffen: 30. Mai 2021

24. Biddle M, Kennedy JW, Wright PM et al (2021) Improving outcomes in acute and chronic periprosthetic hip and knee joint infection with a multidisciplinary approach. Bone Joint Open 2(7):509-514

25. Pattyn C, De Geest T, Ackerman P et al (2011) Preformed gentamicin spacers in two-stage revision hip arthroplasty: functional results and complications. Int Orthop 35(10):1471-1476 
26. Citak M, Luck S, Linke P et al (2021) Manuelle Beimischung von Antibiotika zu industriellen Knochenzementmischungen. Orthopade. https:// doi.org/10.1007/s00132-021-04115-7

27. Mühlhofer HML, Knebel C, Pohlig F et al (2018) Synovial aspiration and serological testing in twostage revision arthroplasty for prosthetic joint infection: evaluation before reconstruction with a mean follow-up of twenty seven months. Int Orthop 42(2):265-271

28. Ghanem M, Schneider I, Zajonz D et al (2021) Management of modular mega-implant infection of the lower extremity.ZOrthop Unfall. https://doi. org/10.1055/a-1340-0890

29. Beaupre LA, StampeK, MassonEetal (2017) Healthrelated quality of life with long-term retention of the prosthesis of antibiotic loaded acrylic cement system following infection resolution in low demand patients. J Orthop Surg (hongkong) 25(2):2309499017716257

30. Petis SM, Perry KI, Pagnano MW et al (2017) Retained antibiotic spacers after total hip and knee arthroplasty resections: high complication rates. JArthroplasty 32(11):3510-3518
Individual megaspacers in two-stage revision of infected total hip arthroplasty - clinical and functional results after 2 years. Individual metal-endoskeleton cement spacer (iMECS)

Background: Large femoral defects in late periprosthetic infection (PPI) after total hip arthroplasty (THA) often require the use of large, individual metal endoskeletonreinforced cement spacers (iMECS).

Objectives: The aim was to record the clinical, radiological and functional results (Harris Hip Score HHS) up to the 2 years after treatment of a PPI using an iMECS. Major patientspecific parameters were to be evaluated with regard to the treatment outcome. Materials and methods: The collective of this single-center retrospective cohort study comprised 29 patients. The mean follow-up was 24.4 months (range: 23.0 to 27.6 months). The absence of infection after endoprosthetic hip joint reconstruction was rated as successful treatment. The two patient groups (successful (S)/not successful $(\mathrm{nS})$ ) were compared with regard to gender distribution, the Charlson comorbidity index $(\mathrm{CCl})$, the number of previous septic changes, and the rate of polymicrobial and difficult-to-treat infections.

Results: The average $\mathrm{CCl}$ in the total collective was 6.4 points. Joint reconstruction was possible in 23 of 29 patients (79\%); 2 years after PPI treatment 4 patients were not available for a follow-up examination ( 2 deceased, 2 unable to participate). At the time of the follow-up, 17 of the 29 patients had received a joint reconstruction and were free of infection, with an average HHS of 75 points. There were no iMECS-associated complications requiring revision. Only the initial $\mathrm{CCI}$ (S: 4.1 points; $n S: 9.7$ points) differed significantly between the patient groups $(p<0.05)$.

Conclusions: In the case of large femoral defects, iMECS provide secure temporary stabilization. The chance of a successful joint reconstruction is closely related to the individual comorbidities profile.

Keywords

Comorbidity - Infection - Reoperation - Total hip replacement - Treatment outcome 
Hier steht eine Anzeige.

黑 Springer 